\title{
Radiation damage to DNA: electron scattering from the backbone subunits
}

\author{
Stefano Tonzani \\ JILA and Department of Chemistry, \\ University of Colorado, Boulder, Colorado 80309-0440 \\ Chris H. Greene \\ Department of Physics and JILA, University of Colorado, Boulder, Colorado 80309-0440
}

(Dated: June 24, 2021)

\begin{abstract}
In the context of damage to DNA by low-energy electrons, we carry out calculations of electron scattering from tetrahydrofuran and phosphoric acid, models of the subunits in the DNA backbone, as a first step in simulating the electron capture process that occurs in the cell. In the case of tetrahydrofuran, we also compare with previous theoretical and experimental data. A comparison of the shape of the resonant structures to virtual orbitals is also performed to gain insight into the systematic connections with electron scattering from similar molecules and dissociative electron attachment experiments.
\end{abstract}

PACS numbers: 31.15.Ew,34.80.-i,34.80.Bm 


\section{INTRODUCTION}

In the past few years, a growing literature has emerged, concerning the damage to nucleic acids by low energy electrons ${ }^{1.2 .3 .4}$ produced by ionizing radiation. The process follows the creation of thousands of low energy electrons, stripped off from molecules in the cell either directly by the radiation or else by its first products, highly energetic primary electrons, that can cause electron-impact ionization. It has been shown that a majority of the secondary electrons have an energy distribution between 0 and $20 \mathrm{eV} \underline{\underline{5}}$ If the electron energy is higher than the ionization threshold for DNA (between 7.85 and $9.4 \mathrm{eV}$, as measured for the DNA bases $\left.^{6}\right)$, then the nucleic acid can be ionized and damage produced through the subsequent rearrangement and dissociation of the cation ${ }^{7}$ If the electron energy is lower, though, damage can still be generated through a negative anion-mediated mechanism, which starts with the capture of the electron in a molecular resonance, followed by a transfer of energy and electron density towards a weak bond that subsequently ruptures. Beyond this generally accepted framework, there are many controversial issues. These issues concern the location of the initial capture site, $\frac{1}{,}$ the dynamics of the metastable anion generated by electron capture and the identification of the final bond that ruptures. ${ }^{2,8}$ The first question is generally answered saying that since the DNA bases have extended conjugated systems, they also have many unoccupied $\pi^{*}$ orbitals, which in turn will give rise to low energy shape resonances.

In a previous article, we have explored the resonances that arise in electron scattering from each of the RNA and DNA bases..$^{\frac{9}{}}$ In the present study, we analyze the behavior of the other subunits in the macromolecule, with the aim of investigating the electron interaction with the moieties that constitute the sugar-phosphate backbone, and verify whether they could play a role in the electron capture stage of the process, In any case, it has been shown ${ }^{10}$ 
that low energy electrons can damage the DNA sugar, deoxyribose (2-deoxy-D-ribose), and this interaction could be important in the cell environment. To model the sugar, we use tetrahydrofuran (THF, whose formula is $\mathrm{C}_{4} \mathrm{H}_{8} \mathrm{O}$ ), which is similar to deoxyribose, except that the latter has - $\mathrm{OH}$ groups attached to the $\mathrm{C}_{1}$ and $\mathrm{C}_{3}$ and a $\mathrm{CH}_{2} \mathrm{OH}$ side chain attached to the $\mathrm{C}_{4}$ (the latter two substituents are linked to a phosphate group in the DNA molecule, while the former is linked to a base), they are substituted here by hydrogens. We use THF instead of deoxyribose because in DNA the $\mathrm{OH}$ groups are fundamentally modified by the phosphate and base that are attached to it (in fact the one attached to the $\mathrm{C}_{1}$ disappears altogether), so we think that the solution would be either to consider a whole nucleotide or use THF which mimics only the ring structure, which is less affected by the rest. Another reason for this choice is that a recent study on dissociative electron attachment (DEA) from all the DNA components 11 has measured the average gas-phase DEA cross section from the bases as being very similar to the cross section per base for supercoiled DNA measured in Ref. 12. This implies the DEA cross section for the sugar and phosphate groups to be much smaller, which is what happens in the case of THF, but not for a hydroxy-substituted THF, for which the DEA cross section is larger than for the bases. This suggests that THF could be a better molecule to model the sugar moiety in DNA. The structures of these compounds are represented schematically in Figs. 1. 2. In practice, the DNA backbone can be thought of approximately as constituted by THF molecules to which the bases are attached, linked together by phosphate groups.

For the phosphate there are no previous results available for comparison, but for THF recently there have been both new experimenta $1^{13,14}$ and theoretical ${ }^{15}$ results, with which we will compare our calculations. We will also attempt a comparison of the resonant wavefunctions to virtual orbitals from a Hartree-Fock calculation, in order to gain some more insights 
into the capture process and its possible consequences on the anion evolution. The conclusions suggested by this analysis lead us to draw a possible link between our calculations and experimental data on dissociative electron attachment .

\section{THEORY}

Electron scattering from a polyatomic molecule is intrinsically a many-body problem that can, under the so-called static-exchange approximation, ${ }^{16}$ be reduced to a one electron problem. The static-exchange approximation amounts to including only the ground state of the target in the close coupling expansion of the wave function, and it is essentially the equivalent of the Hartree-Fock approximation for continuum states. ${ }^{17}$ A detailed description

of our method can be found in Refs. 9, 18, 19, therefore here we will just sketch the main points of the treatment.

To solve this one electron problem, ${ }^{18}$ we use the R-matrix method which starts by partitioning space into two zones: a short range zone where all the channels are coupled and the scattering problem can in principle be treated in all its many body complexity, and an outer zone (external to the target electron density) in which the escaping electron only sees the effect of the target molecule as a multipole expansion of its electrostatic potential. In its eigenchannel form, the R-matrix method can be formulated as a variational principle ${ }^{20}$ for the normal logarithmic derivative $(-b)$ of the wavefunction on the reaction zone surface:

$$
b \equiv-\frac{\partial \log (r \Psi)}{\partial r}=2 \frac{\int_{V} \Psi^{*}(E-\hat{H}-\hat{L}) \Psi d V}{\int_{V} \Psi^{*} \delta\left(r-r_{0}\right) \Psi d V}
$$

where $\hat{L}$ is the Bloch operator, needed to make the Hamiltonian $\hat{H}$ Hermitian and $r_{o}$ is the boundary between the internal and external regions. It is possible, after expanding the 
internal region wavefunction in a suitable basis set, to recast the solution of Eq. 1 as an eigenvalue problem:

$$
\underline{\Gamma} \vec{C}=2(E \underline{O}-\underline{H}-\underline{L}) \vec{C}=\underline{\Lambda} \vec{C} b,
$$

where $\underline{O}$ is a matrix of volume overlap between the basis functions, while $\underline{\Lambda}$ is an overlap of the basis functions on the surface of the internal zone, ${ }^{20}$ essentially the denominator of Eq. 1. and $\underline{H}, \underline{L}$ are the matrix representations of the operators in Eq. 1] Through basis set partitioning we shift the computational burden to the solution of a large linear system. As a basis set we use finite elements ${ }^{21}$ in all three spherical coordinates, in this way we have large but sparse matrices that are amenable for solution with fast sparse solvers. ${ }^{19.22}$

To simplify further the description of our system we have to deal with the nonlocality inherent in the potential. To do this we use a local density approximation for the exchange potential, which reduces it to a functional only of the local density:

$$
V_{e x}(\vec{r})=-\frac{2}{\pi} k_{F} F\left(k_{F}, E\right)
$$

where $k_{F}$ is the local Fermi momentum:

$$
k_{F}(\vec{r})=\left[3 \pi^{2} \rho(\vec{r})\right]^{1 / 3}
$$

and $F$ is a functional of the energy and the local density $\rho(\vec{r})$ (through the local Fermi momentum). The functional form we use for $F$ is called the Hara exchange ${ }^{23}$ It has been extensively employed in continuum state calculations, and it is energy-dependent. The local exchange approximation, widely used also in density functional (DFT) calculations, ${ }^{24}$ has proven itself to give qualitatively correct results, ${ }^{16.18}$ while being sufficiently simple to 
implement computationally that it permits an exploration of complex molecular species.

A polarization-correlation potential is added to this. The long range part of this potential is a simple multipole expansion, of which we retain only the induced dipole polarization term:

$$
V_{p o l}=-\frac{\alpha_{0}}{2 r^{4}}
$$

where $\alpha_{0}$ is the totally symmetric component of the polarizability tensor, and it can be calculated $a b$ initio using electronic structure codes. Exploratory tests suggest that the anisotropic polarizability and the electron-quadrupole interaction are much less important. For example the anisotropic part of the polarizability, when introduced generates a maximum difference of $0.01 \mathrm{rad}$ in the phase shifts, which in turn translates in a variation of the cross section values of roughly $1 \%$.

In the volume where the electronic density of the target is not negligible, this potential is nonlocal. The polarization-correlation interaction can be approximated again as a local potential, different forms of which have been suggested in the literature. The one we use is based on DFT (specifically on the LYP potential of Ref. 25) and it has yielded reliable

results in the work of Gianturco and coworkers. ${ }^{26}$ This form makes use of the electron density, its gradient and Laplacian, which have to be calculated for each target molecule. The short and long range potentials are matched unambiguously (continuously but with discontinuous derivatives) at the innermost crossing point, whose radius is dependent on the angles. The matching is unambiguous in the sense that there are two crossing points between the inner and outer potential for each angle, and we always choose the innermost, since the other is far in the region where the electron density of the molecule is very small. Choosing the outermost crossing has proven to give unphysical results ${ }^{27}$ in many cases. In recent years, 
better density functionals have been devised that give the quantities of interest more directly, without the need for an additional polarization contribution, and they have been recently used in connection with time-dependent DFT to calculate scattering observables. ${ }^{28}$

Since the molecules considered in this work possess dipole moments, we take dipole interaction effects into account in the manner described in Ref. 9. Recently we discovered a mistake in our dipole matching code that led to an overestimation of the cross sections in Fig. 3 of Ref. 9 of about $10 \%$ at very low energy, decreasing and becoming negligible beyond about $10 \mathrm{eV}$, but its effects on the other results in that work are negligible. The quadrupole fields can potentially be significant as well, but they are roughly half of the dipole field at the box boundary; accordingly, we did not include quadrupole interactions outside the box, since their effect was found to be small when analyzed using an R-matrix propagation technique: $\stackrel{29.30}{=}$ The nonspherical part of the polarizability tensor is quite small as well, roughly one fourth of the spherical part, and from previous calculations we have seen how such values have only a minimal influence on the final results; this too has been neglected here.

For molecules with dipole moments the fixed-nuclei scattering cross sections are formally infinite. This divergence can be readily eliminated by considering rotations of the molecule, as a Born closure expansion. ${ }^{31}$ We have not implemented this here, as in our previous work on the DNA bases, because this level of detail is not our present interest. Moreover, the dipole moments here are also less than half what they were for the DNA and RNA bases, so the correction will be even less important here. Therefore the cross sections we show should be understood to include only up to a maximum electronic orbital angular momentum $l_{\max }=10$, with all the higher partial waves omitted. We stress as well that since our model does not include excited states of the target molecule, when the electron energy is above 
any excitation or the ionization threshold (the latter is at roughly $9.8 \mathrm{eV}$ for both molecules in this study, but since our model is approximate we can expect that this will be shifted upward by a few eV), the electron molecule compound will have many more channels to decay into, with the result that the higher energy resonances will be modified by these new interactions.

All the target quantities are calculated at the Hartree-Fock level using a $6-31 \mathrm{G}^{* *}$ basis set, and the target equilibrium geometries have been optimized at the same level of theory. The remaining details of the calculations are very similar to those reported in Ref. 9, including the convergence criteria. The dimension of the matrices is roughly 200000 by 200000, and our convergence studies show that increasing the number of sectors by $30 \%$ lowers the position of the resonances by about $0.1 \mathrm{eV}$ in THF. These calculations are very cumbersome, and for the level of accuracy we are aiming for here this convergence criterion seems adequate. For details on the treatment of the integrals and of the nuclear Coulomb singularities we refer the reader to our previous publications, specifically Sec. IIE of Ref. 18 and Sec. IID of Ref. 9 .

\section{RESULTS: THF}

In Fig. 3] our results are compared to the low-resolution experimental data of Zecca et $a l .13$ and the theoretical results of Bouchiha et al., $\frac{15}{15}$ obtained like ours without performing a Born closure to consider the effect of the dipole field on the higher partial waves. We also plot the data in the form of a time-delay, ${ }^{\frac{9}{3}}$ to make the resonance position and width more evident. The total time-delay is the sum of the eigenvalues of the hermitian matrix

$$
Q=i S \frac{d S^{\dagger}}{d E}
$$


where $S$ is the scattering matrix. In Fig. 3 we also plot the resonant channels, particular eigenvalues of the time-delay matrix that are larger than the others and show a Lorentzian behavior that sets them apart from the rest. We rescaled the total time-delay by dividing it by a factor, to show more easily everything in the same graph.

The order of magnitude of our cross section is not different from the results of Ref. 15 calculated without Born closure. Since our calculations do not include excited states of the target, we will not be able to detect core-excited resonances (present instead in the calculations of Ref. 15). Also comparing with total cross sections we will be missing rotational and vibrational excitations and the electronically inelastic channels, therefore elastic cross sections should be lower than experimental total cross sections, this does not happen in our case, and it is partly due to the approximate nature of our model. With respect to experiment, the comparison is difficult due to the effect of higher partial waves (with $l>10$ ). The Born correction has been calculated in Ref. 15, and the cross section becomes higher than experiment, we would expect a similar effect for our data. Due to the low resolution, the experimental results below $5 \mathrm{eV}$ are (in the words of the authors) to be considered only indicative. The resonant structure that appears around $9.3 \mathrm{eV}$ in our results looks similar to the analogous experimental feature, also taking into account that our model usually predicts resonances about 1.5-2 $\mathrm{eV}$ higher than their experimental position, as verified for the DNA bases $\underline{9}$ and also for molecules like carbon dioxide, benzene and $\mathrm{SF}_{6}, \stackrel{32}{\underline{3}}$ because of the approximations adopted. This is due mostly to the adoption of a local exchange model, since for example, in a system like $\mathrm{CO}_{2}{ }^{33.34}$ this approximation causes a resonance shift of around $2 \mathrm{eV}$ or so for model static-exchange, compared to all-electron static-exchange calculations, that is not possible to compensate with the polarization-correlation potential, which indeed shifts the resonance by a similar amount for this molecule (1.5 eV compared to 
$2 \mathrm{eV}$ ) with respect to correct polarization. Also the model polarization is approximate, but the model exchange constitutes the largest error in our calculations. Indeed, if we perform a purely static-exchange calculation (see Fig. 3) the position of the resonance (maximum of the time-delay) is at $11.9 \mathrm{eV}, 2.6 \mathrm{eV}$ higher than the result including polarization, and it is composed of three overlapping resonances. For what we said above about the shifts generated by the model potential with respect to exact exchange, this resonance should be around $10 \mathrm{eV}$ in an all-electron calculation, therefore probably visible in the calculations of Ref. 15 that go up to $10 \mathrm{eV}$ in energy. When polarization-correlation is added this value should fall below $10 \mathrm{eV}$, and thus be clearly visible in the data of Ref. 15 .

From the fact that our resonances are shifted too high in energy one might have thought that the widths would turn out to be larger than the experimental ones. We have verified in the past for many systems $\$ .32$ that our calculated widths are comparable to their correct values.

No resonance was found in this energy range in the other published theoretical study $\underline{\underline{15}}$ Through a Lorentzian fit, we predict a width of about $2.5 \mathrm{eV}$ for this resonance. At the static-exchange level, the resonance has a larger width, of about $3.1 \mathrm{eV}$. A higher energy resonance is found at $16.2 \mathrm{eV}$ and it is even broader (about $3.0 \mathrm{eV}$ ). The partial wave contributions are mainly $l=4$ (with a contribution of $70 \%$ ) for the lower energy resonance and for the higher energy resonance $l=5(50 \%)$ and $l=3(30 \%$, as can be seen in Tab. II). The partial wave decomposition will depend somewhat on what center in chosen as the expansion. We always perform the decomposition around the center of mass of the molecule, which seems a reasonable choice.

To understand better the reason for the difference between our results and the ones in Ref. 15 and to make sure that our results are consistent with other known results, we have 
first of all performed a time-delay analysis ${ }^{20.35}$ of our scattering data, shown on the bottom of Fig. 3. This has allowed us to establish the presence of three superimposed resonances, two of which peak at $9 \mathrm{eV}$ (with one sensibly more intense than the other) while the third peaks around $10 \mathrm{eV}$, and its intensity falls between the other two. Due to the large width of these resonances, the weakest of the channels in Fig. 3 has a time-delay eigenvalue which is not much larger than the nonresonant eigenvalues.

An existing calculation for cyclopropane ${ }^{36}\left(\mathrm{C}_{3} \mathrm{H}_{6}\right)$ shows that this cycloalkane has a cross section with a similar resonant structure at $6 \mathrm{eV}$. It is superimposed on a broader resonance at higher energy and other nonresonant contributions that give a very wide plateau, although in this case the first feature is not a composite resonance. The symmetry of the cyclopropane resonance in Ref. 36 is even with respect to the plane of the three carbon atoms, and the same occurs in THF, in the sense that although this compound is not planar, our resonance structures do not change sign above and below the ring bonds. It is plausible then that due to its larger frame and density of virtual orbitals, THF is able to support more quasi-degenerate resonant states at a similar energy.

Since there are no other existing experimental data on THF, we have performed calculations for cyclopentane $\left(\mathrm{C}_{5} \mathrm{H}_{10}\right)$, which is very similar to THF except that here the oxygen atom is substituted with a $-\mathrm{CH}_{2^{-}}$group. For this molecule, experimental data ${ }^{37}$ shows a broad shape resonance at $8 \mathrm{eV}$. The similarity of the two molecules suggests that many features of the cross section will be analogous. The results are compared to THF in Fig. 4. The cross section closely resembles that of $\mathrm{THF}$, with a triple resonance superimposed on a broad higher energy resonance centered around $16 \mathrm{eV}$, which produces a wide plateau. The electron scattering cross sections for alkanes and cycloalkanes of different sizes have very similar behaviors, ${ }^{37.38}$ namely the same resonant structures and a magnitude dependent on 
the number of carbon atoms. Our evidence thus suggests that THF, being very similar to a cycloalkane, shares these common features, as suggested also in Ref. 13. Also the cross section for cyclopentane is, in our calculations, larger than in THF, a trend that seems to be confirmed by the experimental data. ${ }^{13.38}$ The cyclopentane cross section peak is roughly $50 \AA^{2}$ which is very close to the experimental value ${ }^{37}$ for the total cross section. Recent and as yet unpublished theoretical calculations, ${ }^{39}$ performed on a THF molecule distorted to achieve a planar geometry for the furanose ring, show results similar to ours, with a double shape resonance in a similar energy range.

As in Ref. 9 we attempt to correlate the spatial shape of the resonant wavefunction with the Hartree-Fock virtual orbitals obtained with a small $\left(6-31 \mathrm{G}^{* *}\right)$ basis set. In this case the analysis is complicated by the fact that the molecules are not planar, so a projection on a twodimensional surface would not pass through all the nuclei and, since the resonance is wide, different contributions mix and overlap. However, we have established that the resonant wavefunction at its peak resembles closely a virtual orbital with energy of $7 \mathrm{eV}$ (orbital 23). At higher energy (around $10.5 \mathrm{eV}$, when the second resonant contribution becomes dominant), the resonance spatial structure appears similar to an orbital of $11 \mathrm{eV}$ of energy (orbital 31). We show this in Fig. 5. Since there is a great degree of resonance overlap, the relationship of the resonant wavefunctions to the virtual orbitals we show becomes even clearer when observing the other virtual orbitals, which are actually very different from our wavefunctions.

Recently, DEA cross sections have been measured ${ }^{11}$ for all the DNA subunits. These cross sections display two prominent resonances at 6 and $8 \mathrm{eV}$, of width around $1 \mathrm{eV}$ each for THF, which could correlate with the calculated shape resonance, although clearly much complicated vibrational dynamics happens between electron capture and dissociation that 
we do not take into account here, and in the results of Ref. 15 there are electronically inelastic resonances in this energy region which could lead to similar outcomes in DEA.

Several electron-energy-loss (EEL) studies of $\mathrm{THF}^{40.41}$ and cyclopentane ${ }^{42}$ have been performed, and some attempts have been made at assigning the resonances, also in relation to the position of the excited states from absorption spectroscopy. In Ref. 41 a gas-phase EEL peak for THF at $6.6 \mathrm{eV}$ is assigned as a core-excited resonance with a parent ion of Rydberg character, centered on the oxygen atom, whereas a similar experimental peak in cyclopentane in Ref. 42 is assigned as a shape resonance, on the basis of a molecular orbital argument, with the electron wavefunction distributed around the whole ring. Our calculations seem to agree with this second assignment, for two reasons: first, the resonance is very similar in character in THF and cyclopentane (see Fig. 4), and therefore it seems implausible that it derives from excitations on the oxygen; second, the resonant wavefunctions in Fig. [5] are found to be delocalized over the entire ring as had been speculated in Ref. 42 for cyclopentane. At higher energies core-excited contributions are most likely predominant, as was suggested in Ref. 40.

It is difficult to attempt a prediction of what the evolution of the resonances found in elastic scattering could be, when the nuclear dynamics is included. At present this is computationally prohibitive, but a first analysis can be attempted by inspecting our calculated results. From the spatial structures of the resonant wavefunctions it seems likely that the resonances could initiate a break-up of the ring, because of the presence of many nodal surfaces cutting through the ring bonds. For example, the experimental DEA cross sections for deoxyribose in Ref. 10 exhibit a peak at around $6 \mathrm{eV}$ for production of the anion $\mathrm{C}_{3} \mathrm{H}_{5} \mathrm{O}_{3}^{-}$, which could be generated by a resonant structure that shows a node cutting through the ring from $\mathrm{C}_{3}$ to $\mathrm{C}_{1}$, similar to the top left plot in Fig. 5, given that deoxyribose 
has side groups attached to $\mathrm{C}_{4}$ and $\mathrm{C}_{3}$ that could lead to production of the aforementioned anion.

\section{RESULTS: $\mathrm{H}_{3} \mathrm{PO}_{4}$}

In the case of phosphoric acid there are no available theoretical or experimental data to compare with, and consequently our analysis of the results will be somewhat more limited. The cross sections show two prominent resonances, one at $9.1 \mathrm{eV}$ and the other at $15.8 \mathrm{eV}$, with respective widths $2.0 \mathrm{eV}$ and $1.5 \mathrm{eV}$. The main partial wave contributions are $l=4$ and $l=3$ at $40 \%$ and $30 \%$ respectively for both resonances, as stated in Tab. I At low energies there is a sharp rise in cross section due to the dipole moment. Cross section and total time delay are illustrated in Fig. [6. It is difficult to say why the second resonance is narrower than the first, since this is somewhat counterintuitive. We will limit ourselves to pointing out that in a complicated polyatomic molecule, shape resonance widths depend not only on the total energy, but also the size of the probability density near the escape regions in the multidimensional potential. It is possible, therefore, that a higher-energy resonance could be narrower than one at lower energy. Resonances broader at lower energy than the higher energy ones can be seen, for example, in $\mathrm{C}_{60}$ fullerene. ${ }^{26.43}$

In Ref. 11 the DEA cross section has been measured for trimethylphosphate $\left(\mathrm{PO}_{4}\left(\mathrm{CH}_{3}\right)_{3}\right)$, and it displays a very wide and prominent resonant structure at $7.5 \mathrm{eV}$, to which our 9.1 $\mathrm{eV}$ resonance could correlate. No branching ratio has been measured for this compound. The structure of phosphoric acid presents three identical -OH groups and therefore the resonances will show structures with a similar probability for the scattering electron to end up in each of these groups, making for complicated spatial profiles, which in the end are not very illuminating. For these reasons we will not attempt here an analysis of the spatial 
structure of the resonances.

As in THF, the shape resonances are quite broad, so the anions will be relatively short lived, and probably they will autoionize back (possibly with vibrational excitation) before having the possibility to lead to a breakup or energy transfer to another DNA subunit. However, the presence of water, a structural component in biological DNA, can act to stabilize the anions, and probably will significantly influence the lifetimes of these resonances in the cell nucleus, since the backbone is in closer contact with the water and the surrounding environment, compared to the bases that lie inside the double helix and are presumably somewhat less affected by the presence of the solvent.

\section{CONCLUSIONS}

We have calculated electron scattering cross sections from molecules that closely resemble the DNA sugar-phosphate backbone components, namely tetrahydrofuran and phosphoric acid, as a first step to model the DNA radiation damage caused by low energy electrons. Since our model is approximate, resonance shifts of around $2 \mathrm{eV}$ higher than experiment are usually observed. In THF we find a very broad resonance, which agrees (within the limits of our model) with recent experimental data, but not with one recent theoretical calculation. Also for phosphoric acid we find shape resonances. In both cases the resonances are quite broad and high in energy and are not expected to play a major role, compared to those of the nitrogenous bases, in electron capture leading to damage in DNA. For THF we have also attempted to correlate the resonant structures with recent DEA experiments and to electron scattering experiments from other related molecules. 


\section{Acknowledgments}

This work was supported by the Department of Energy, Office of Science, and NERSC with an allocation of supercomputing resources. We thank P. Burrow, J. Gorfinkiel, L. Sanche and L. Caron for stimulating discussions, the group of J. Toomre for computational time on their machines and N. Mehta for the use of his R-matrix propagator computer code.

1 B. Boudaiffa, P. Cloutier, D. Hunting, M. A. Huels, and L. Sanche, Science 287, 1658 (2000).

2 Y. Zheng, P. Cloutier, D. J. Hunting, J. R. Wagner, and L. Sanche, J. Chem. Phys. 124, 064710 (2006).

3 A. Scheer, K. Aflatooni, G. A. Gallup, and P. D. Burrow, Phys. Rev. Lett. 92, 068102 (2004).

4 S. Ptasinska, S. Denifl, S. Gohlke, P. Scheier, E. Illenberger, and T. D. Mark, Ang. Chem. Int. Ed. 45, 1893 (2006).

5 V. Cobut, Y. Fongillo, J. P. Patau, T. Goulet, M.-J. Fraser, and J.-P. Jay-Gerin, Radiat. Phys. Chem. 51, 229243 (1998).

6 S. G. Lias, R. D. Levin, and S. A. Kafafi, in NIST Chemistry WebBook, NIST Standard Reference Database Number 69, edited by P. Linstrom and W. Mallard (National Institute of Standards and Technology, Gaithersburg, MD, 2005), http://webbook.nist.gov.

7 A. F. Fuciarelli and J. D. Zimbrick, eds., Radiation Damage in DNA: Structure/Function Relationships at Early Times (Battelle, Columbus, OH, 1995).

8 R. Barrios, P. Skurski, and J. Simons, J. Phys. Chem. B 106, 7991 (2002).

9 S. Tonzani and C. H. Greene, J. Chem. Phys. 124, 054312 (2006).

10 S. Ptasinska, S. Denifl, P. Scheier, and T. D. Mark, J. Chem. Phys. 120, 8505 (2004). 
11 K. Aflatooni, A. Scheer, and P. D. Burrow, Phys. Rev. Lett. submitted (2006).

12 B. Boudaiffa, P. Cloutier, D. Hunting, M. A. Huels, and L. Sanche, Radiat. Res. 157, 227 (2002).

13 A. Zecca, C. Perazzolli, and M. J. Brunger, J. Phys. B 38, 2079 (2005).

14 A. R. Milosavljevic, A. Giuliani, D. Sevic, M. J. Hubin-Franskin, and B. Marinkovic, Eur. Phys. J. D 35, 411 (2005).

15 D. Bouchiha, J. D. Gorfinkiel, L. G. Caron, and L. Sanche, J. Phys. B 39, 975 (2006).

16 M. Morrison and L. A. Collins, Phys. Rev. A 17, 918 (1978).

17 C. Froese-Fischer, T. Brage, and P. Jonsson, Computational atomic structure (Insitute of Physics, Bristol, 1997).

18 S. Tonzani and C. H. Greene, J. Chem. Phys. 122, 014111 (2005).

19 S. Tonzani, Comp. Phys. Comm. submitted (2006).

20 C. H. Greene, M. Aymar, and E. Luc-Koenig, Rev. Mod. Phys. 68, 1015 (1996).

21 Bioengineering Institute, The University of Auckland, New Zealand, Fem/bem notes (1997), http://www.bioeng.auckland.ac.nz/cmiss/fembemnotes/fembemnotes.pdf.

22 Scientific Computing Group, University of Basel, Pardiso, http://www.computational.unibas.ch/cs/scicomp/software/pardiso/.

23 S. Hara, J. Phys. Soc. Jpn. 27, 1009 (1969).

24 R. G. Parr and W. Yang, Density Functional Theory of Atoms and Molecules (Oxford University Press, Oxford, 1989).

25 C. Lee, W. Yang, and R. G. Parr, Phys. Rev. B 37, 785 (1988).

26 R. R. Lucchese, F. A. Gianturco, and N. Sanna, Chem. Phys. Lett. 305, 413 (1999).

27 F. A. Gianturco and J. A. Rodriguez-Ruiz, Phys. Rev. A 47, 1075 (1993). 
28 A. Wasserman, N. T. Maitra, and K. Burke, J. Chem. Phys. 122, 144103 (2005).

29 K. L. Baluja, P. G. Burke, and L. A. Morgan, Comp. Phys. Comm. 27, 299 (1982).

30 N. P. Mehta, B. D. Esry, and C. H. Greene, unpublished (2006).

31 F. A. Gianturco, S. Meloni, P. Paioletti, R. R. Lucchese, and N. Sanna, J. Chem. Phys. 108, 4002 (1998).

32 S. Tonzani and C. H. Greene, unpublished (2005).

33 N. F. Lane, Rev. Mod. Phys. 52, 29 (1980).

34 F. A. Gianturco and T. Stoecklin, J. Phys. B 29, 3933 (1996).

35 F. T. Smith, Phys. Rev. 118, 349 (1960).

36 R. Curik and F. A. Gianturco, J. Phys, B 35, 717 (2002).

37 M. Kimura, O. Sueoka, A. Hamada, and Y. Itikawa, Adv. Chem. Phys. 3, 537 (2000).

38 O. Sueoka, C. Makochekanwa, H. Tanino, and M. Kimura, Phys. Rev. A 72, 042705 (2005).

39 C. N. Trevisan and A. E. Orel, unpublished (2006).

40 M. Lepage, S. Letarte, M. Michaud, F. Motte-Tollet, M.-J. Hubin-Franskin, D. Roy, and L. Sanche, J. Chem. Phys. 109, 5980 (1998).

41 L. J. Bremner, M. G. Curtis, and 1. C. Walker, J. Chem. Soc. Faraday Trans. 87, 1049 (1991).

42 M. Allan and L. Andric, J. Chem. Phys. 105, 3559 (1996).

43 C. Winstead and V. McKoy, Phys. Rev. A 73, 012711 (2006).

\section{Figures}




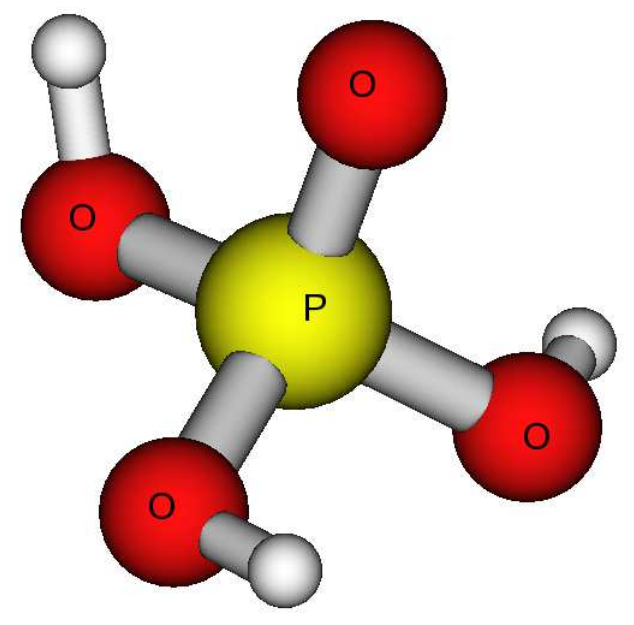

Phosphoric acid

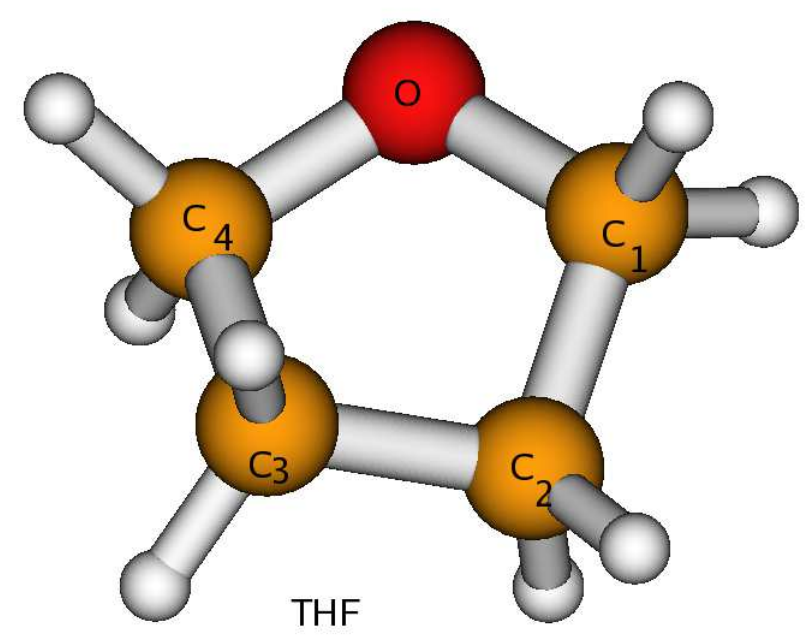

19 
FIG. 1: (Color online) Three dimensional structures of phosphoric acid and tetrahydrofuran. The small circles are hydrogen atoms. 


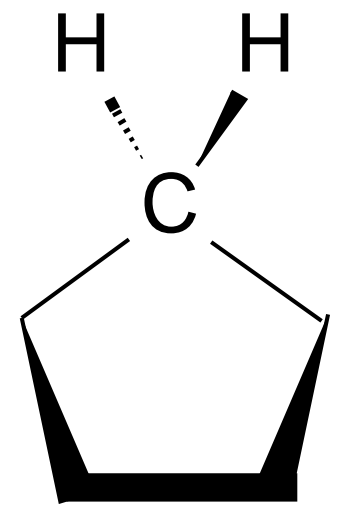

(I)

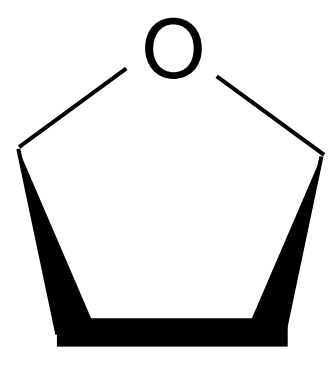

(II)
$\mathrm{OH}$

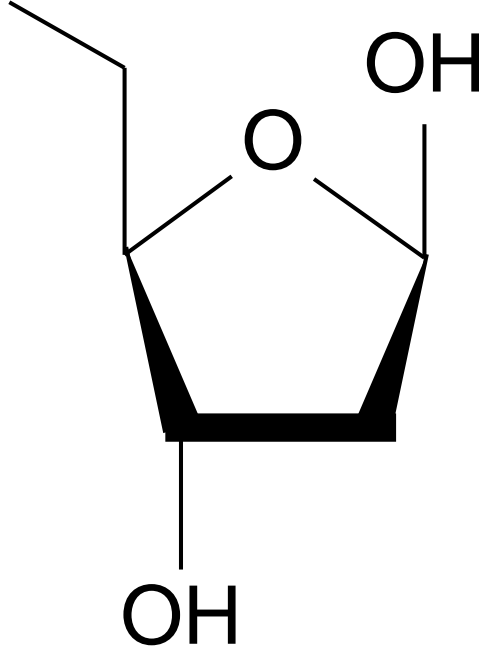

(III) 
FIG. 2: Schematic structures of cyclopentane (I), THF (II), and the DNA sugar deoxyribose (III), that show the similarities between these compounds. The hydrogen atoms that fill the carbon valences are not shown. The ring conformation we have used in the calculations is not planar but puckered, as indicated in Fig. [1 for THF, therefore the $\mathrm{C}_{2}$ carbon is below the plane and $\mathrm{C}_{3}$ above, here they are indicated both above for ease of drawing. 

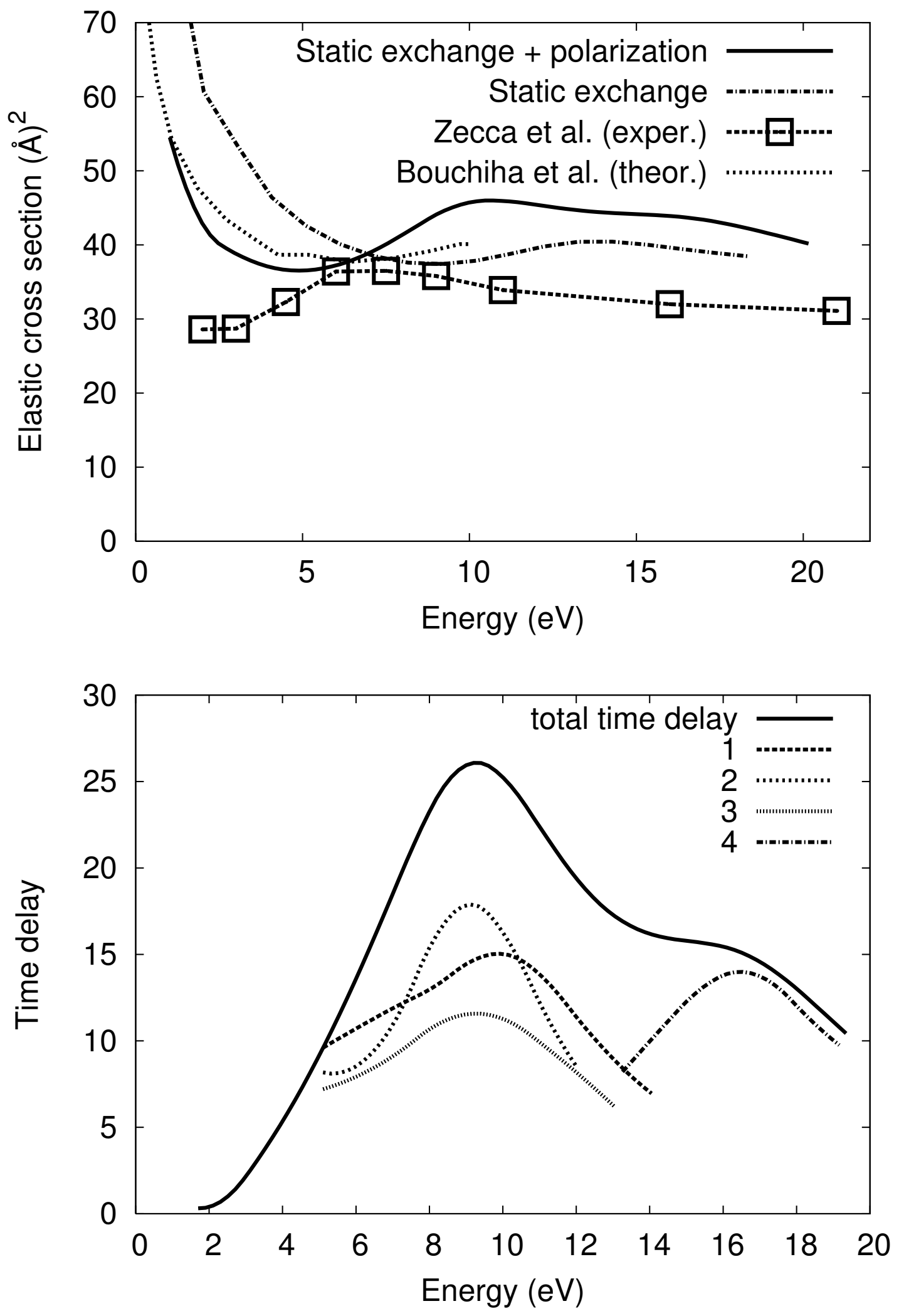
FIG. 3: Partial elastic electron scattering cross sections from THF (solid line), the dot-dashed line represents the static-exchange results. Calculations involve partial waves up to $1=10$ and the dipole physics outside the R-matrix box is included exactly for those partial waves. Top: cross section comparison with theoretical (dotted) $\stackrel{15}{\underline{15}}$ and experimental (dashed) $\stackrel{13}{\underline{13}}$ results, the open squares are the actual experimental data points. Bottom: time-delay plot to highlight the presence of resonances. The full curve is the total time delay, while the numbered curves correspond to the few highest eigenvalues that exhibit resonant behavior. The total time delay was rescaled to show all curves on the same graph more easily. 


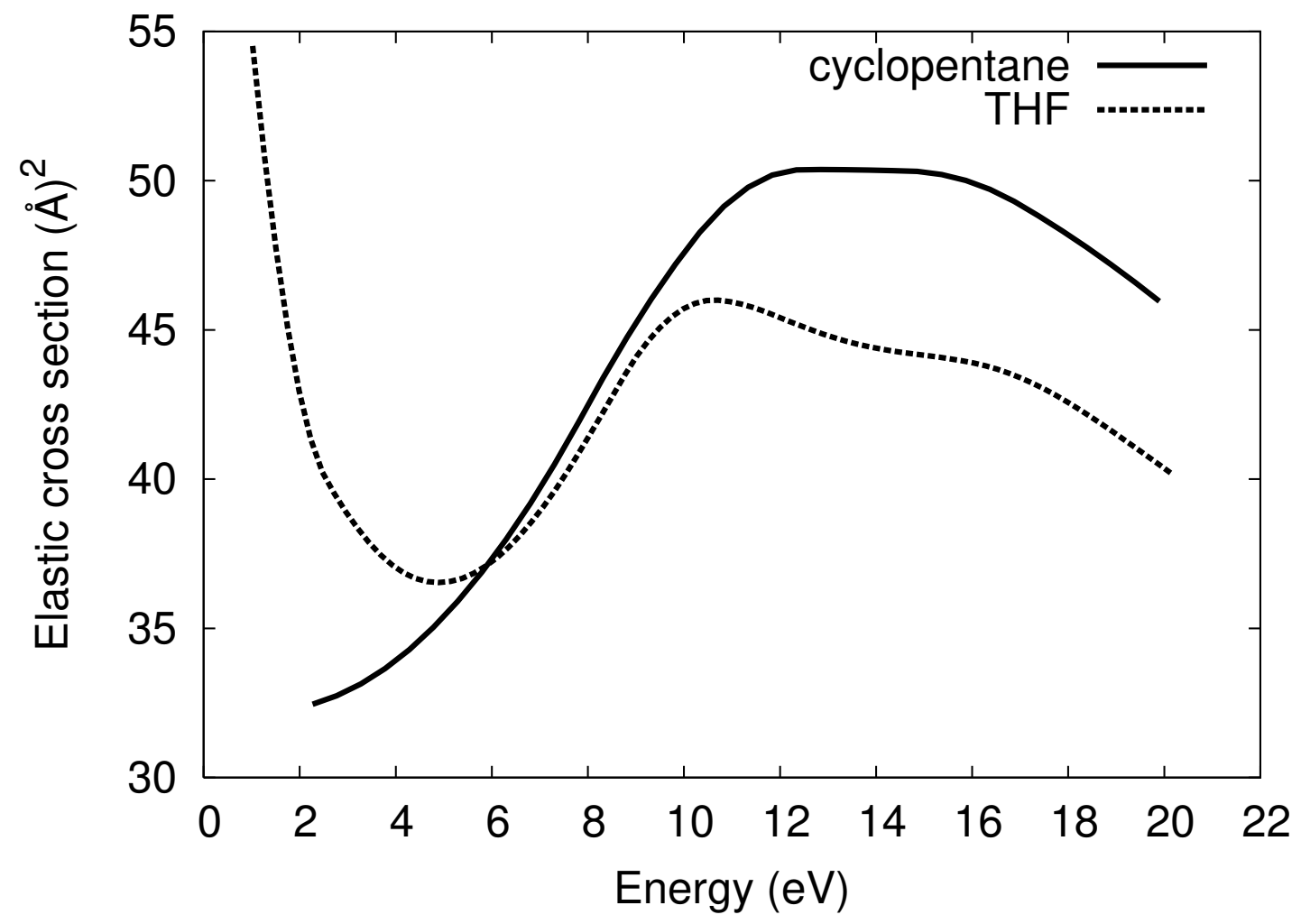


FIG. 4: Unconverged fixed nuclei elastic cross sections for electron scattering by THF and cyclopentane. The two molecular structures are similar (THF has an oxygen atom instead of a $-\mathrm{CH}_{2}{ }^{-}$ group) and so are their electron scattering cross sections. Cyclopentane has essentially zero dipole moment, therefore the low energy part of its cross section does not rise the way it does in THF. 

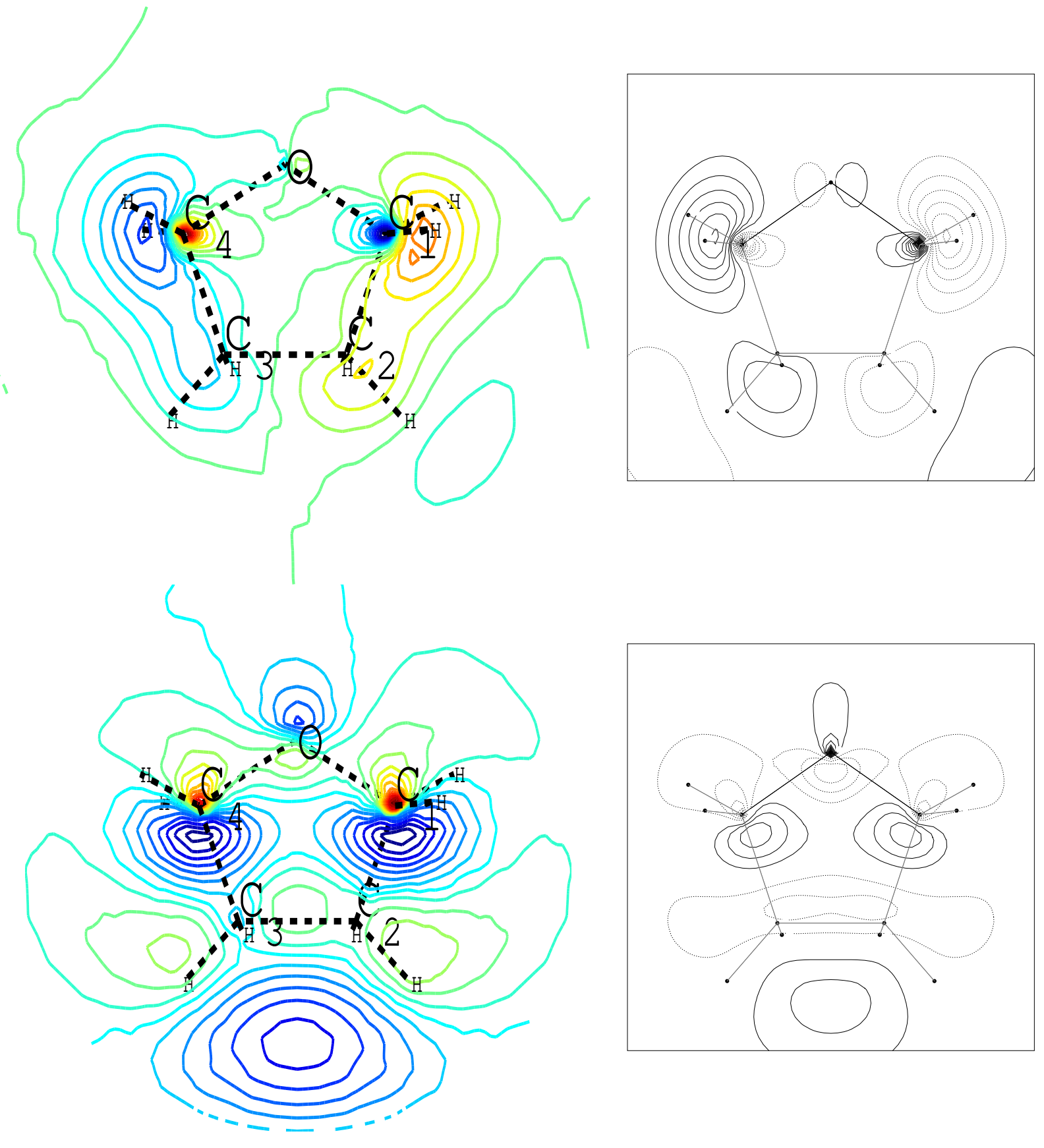
FIG. 5: (Color online) Left: Time delay eigenfunctions for THF on resonance at 9eV (top, where the widely-spaced dotted curve (labeled "2") in Fig. 3 is dominant) and 10.5eV (bottom, where instead the dashed curve (labeled "1") in Fig. 3 dominates). A slice of the three dimensional eigenfunctions is shown, on the plane that contains $\mathrm{OC}_{1} \mathrm{C}_{4}$, while $\mathrm{C}_{3}$ is above the plane and $\mathrm{C}_{2}$ below. The red contours identify positive areas and the blue negative areas of the real part of the wavefunction. Right: virtual orbitals for energies around 7 (top) and $11 \mathrm{eV}$ (bottom) from HF at the $6-31 \mathrm{G}^{* *}$ level. The full lines identify positive areas and the broken lines negative areas. The correspondence between the two top plots and the two bottom plots is very pronounced, allowing to identify the two main contributions to the resonance. Although the molecule is not planar this projection is much easier to read than the three dimensional structures. 

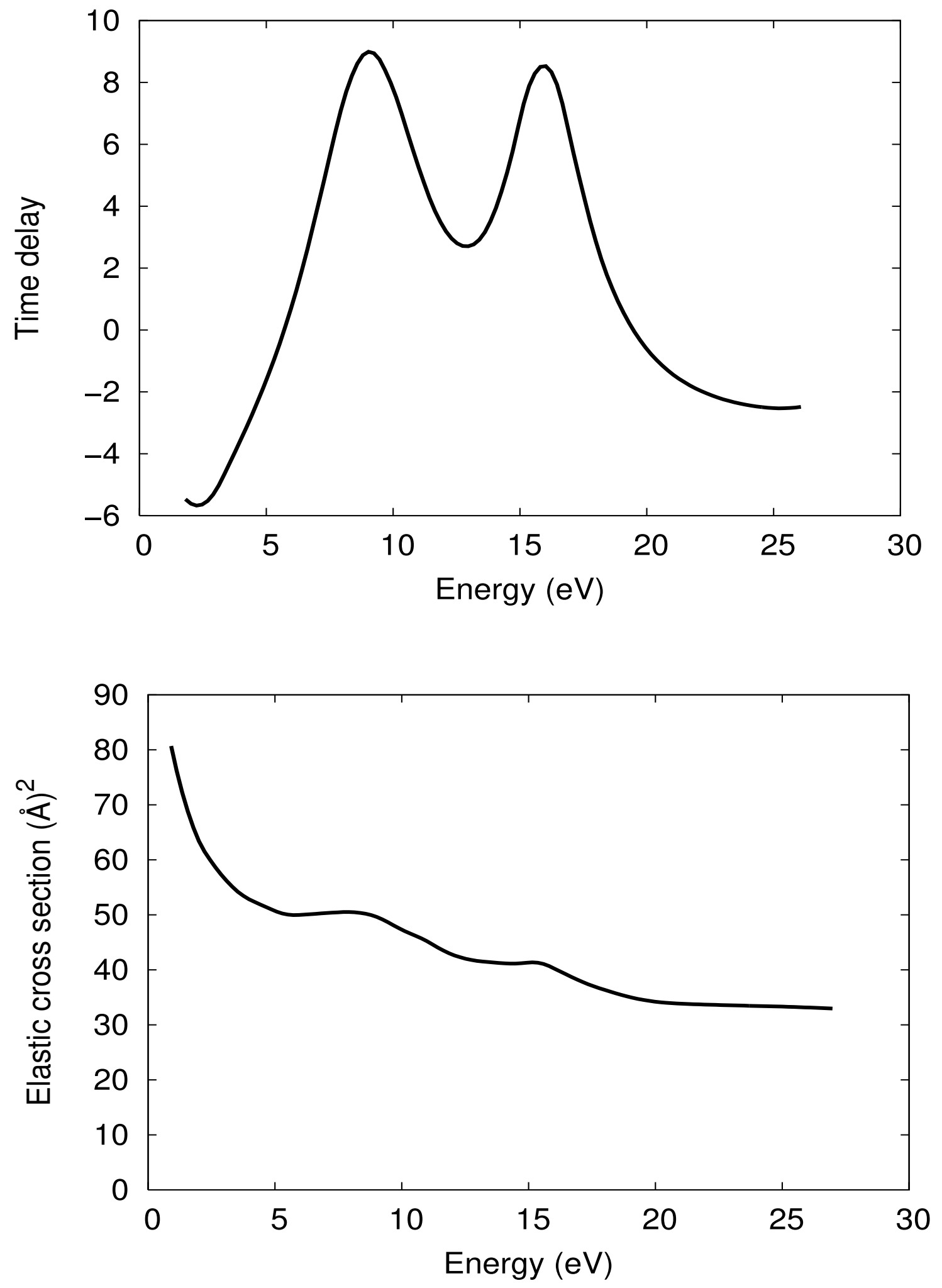
FIG. 6: $\mathrm{H}_{3} \mathrm{PO}_{4}$ partial elastic cross section (top) and time-delay analysis (bottom). Calculations again involve partial waves up to $l=10$ and the dipole physics outside the R-matrix box is included exactly for those partial waves. Two broad resonances are present, at 8.9 and $15.7 \mathrm{eV}$, the cross section is smaller than in the DNA bases and comparable to THF.

\section{Tables}




\begin{tabular}{|l|c|c|c|}
\hline Molecule & Energy (eV) & Width (eV) & Partial wave \\
\hline THF & 9.3 & 2.5 & $4(70 \%)$ \\
& 16.2 & 3.0 & $5(50 \%)$ \\
\hline $\mathrm{H}_{3} \mathrm{PO}_{4}$ & 9.1 & 2.0 & $4(42 \%)$ \\
& 15.9 & 1.5 & $4(41 \%)$ \\
\hline
\end{tabular}


TABLE I: Energies, widths and dominant partial waves of the resonances discussed in the text. 